\title{
INFINITE QUASI-INJECTIVE GROUPS
}

\author{
by M. J. TOMKINSON
}

(Received 10th October 1986)

\section{Introduction}

A group $G$ is said to be quasi-injective if, for each subgroup $H$ of $G$ and homomorphism $\theta: H \rightarrow G$, there is an endomorphism $\bar{\theta}: G \rightarrow G$ such that $\left.\bar{\theta}\right|_{H}=\theta$. It is of course well known that the category of groups does not possess non-trivial injective objects and so we consider groups satisfying the weaker condition of quasi-injectivity.

The quasi-injective abelian groups are determined in [2, p. 106]; these are either divisible abelian groups or are periodic and each Sylow $p$-subgroup is a direct product of isomorphic cyclic or quasicyclic groups. The quasi-injective finite groups were completely characterized by Bertholf and Walls [1]. They showed in particular that every subgroup of a finite quasi-injective group is a T-group (i.e., every subnormal subgroup is normal) and that finite quasi-injective groups are supersoluble and metabelian.

We do not attempt to give a complete characterization of infinite quasi-injective groups as there will be very considerable difficulties in dealing with certain types of infinite group. Ol'šanskii [5] has constructed infinite groups in which every proper subgroup has prime order and any two subgroups of the same order are conjugate. Such a group $G$ would be quasi-injective if, for each $x \in G$, and for each integer $n$, the mapping of $x$ to $x^{n}$ could be extended to an automorphism of $G$. It is not clear to the author whether this can, in fact, be done but it seems likely that some variation on Ol'šanskiî's construction would lead to a quasi-injective group.

To exclude the possibility of such examples arising, we restrict our investigations to more manageable classes of groups, namely soluble groups and locally finite groups. In these cases we are able to give a characterization of the quasi-injective groups which is similar to that in the finite case. In particular, we shall see that quasi-injective groups which are soluble or locally finite are T-groups. Strangely, we have been unable to give a direct proof of this fact for nonperiodic groups but the arguments used in this case are related to those used by Robinson [6] in his investigations of infinite T-groups.

Our results may be summarized in the following theorem.

Theorem. (A) A nonperiodic soluble group is quasi-injective if and only if it is a divisible abelian group.

(B) A locally finite group $G$ is quasi-injective if and only if it is one of the following two types:

(I) $G=Q \times G_{1}$, where $Q$ is the quaternion group of order 8 and $G_{1}$ is a quasi-injective $2^{\prime}$-group. 
(II) $G$ is a split extension of a normal $\pi^{\prime}$-group $K$ by a $\pi$-group $H$ satisfying the conditions:

(i) $H$ and $K$ are abelian groups in which each Sylow subgroup is a direct product of isomorphic cyclic or quasi cyclic groups,

(ii) $K=G^{\prime}$,

(iii) every maximal $\pi$-subgroup of $G$ is a complement to $K$,

(iv) for each $h \in H, p \in \pi^{\prime}$ and positive integer $r$, there is an integer $m=m(p, r, h)$ such that $h^{-1} a h=a^{m}$, for every element $a \in K$ having order dividing $p^{r}$,

(v) if $\sigma \subseteq \pi$ and $K_{\sigma}$ is the Sylow $\sigma$-subgroup of $K$, then $C_{H}\left(K_{\sigma}\right)$ is a direct factor of $H$.

Part (B) of this theorem is very similar to the finite case. There is a slight adjustment to condition (iv) to allow for quasi cyclic groups to appear in $K$ but the significant change is the addition of condition (iii) requiring that every maximal $\pi$-subgroup is a complement to $K$. In particular, this implies that any two maximal $\pi$-subgroups are conjugate under some automorphism of $G$ (see, for example [3, Hilfssatz VI.7.14]). This seems to be a very strong condition which would repay further investigation. Certainly it does prevent some simple constructions from providing examples of quasi-injective groups.

For example, let $K=D r_{i=1}^{\infty} X_{i}$, where $X_{i}=\left\langle x_{i}\right\rangle$ is cyclic of prime order $p_{i}$, where the $p_{i}$ are distinct odd primes. If $H=D r_{i=1}^{\infty} Y_{i}$, where $Y_{i}=\left\langle y_{i}\right\rangle$ has order 2 and we form the split extension $G=K H$ in which

$$
\begin{gathered}
y_{i} x_{i} y_{i}=x_{i}^{-1}, \\
y_{i} x_{j} y_{i}=x_{j} \quad(j \neq i),
\end{gathered}
$$

then $G$ is quasi-injective. The group $G$ is just the direct product of the finite groups $\left\langle x_{i}, y_{i}\right\rangle$ and so the maximal 2-subgroups are just the subgroups which are locally conjugate to $H$ in $G$.

However, if we let $\bar{H}=\prod_{i=1}^{\infty}\left\langle y_{i}\right\rangle$ and form $\bar{G}=K \bar{H}$, then $\bar{G}$ does not satisfy condition (iii) as the subgroup $\left\langle x_{1} y_{1}, x_{2} y_{2}, \ldots\right\rangle$ is a maximal 2-subgroup which, being countable, clearly does not complement $K$.

In fact, we have been unable to construct any quasi-injective group $G=K H$ in which $H / C_{H}(K)$ is uncountable and would conjecture that this situation can not occur.

But even the assumption of countability of $H$ does not ensure that $G=K H$ satisfies condition (iii). If we take $H_{0}=\left(D r_{i=1}^{\infty} Y_{i}\right) \times\langle y\rangle$ where $y$ has order 2 and $y x_{i} y=x_{i}^{-1}$, for all $i$, then in the group $G_{0}=K H_{0}$ we again have $L=\left\langle x_{1} y_{1}, x_{2} y_{2}, \ldots\right\rangle$ as a maximal 2-subgroup but $|G: K L|=2$.

\section{Soluble groups}

Although some of the results here apply to all soluble quasi-injective groups and will be used later, our main aim in this section is to determine the nonperiodic soluble quasi-injective groups. The remaining soluble groups will then be included in the locally finite case which is dealt with in Section 3. 
The first three parts of the following lemma are given in Bertholf and Walls [1]. The results on fully invariant subgroups will be most useful when applied to terms of the derived series.

Lemma 2.1. (i) $A$ direct factor of a quasi-injective group is quasi-injective.

(ii) A fully invariant subgroup of a quasi-injective group is quasi-injective.

(iii) If a fully invariant subgroup of a quasi-injective group has an element of order $n$ (including $n$ infinite) then it contains all elements of order $n$.

(iv) If the quasi-injective group $G$ has an element of infinite order then $G$ is radicable.

Proof. (iv) Let $x \in G$ have infinite order and let $g$ be any element of $G$. If $n$ is a positive integer, consider the homomorphism $\theta:\left\langle x^{n}\right\rangle \rightarrow\langle g\rangle$ which maps $x^{n}$ onto $g$. This mapping extends to an endomorphism $\bar{\theta}: G \rightarrow G$. If $x \bar{\theta}=y$, then

$$
y^{n}=(x \bar{\theta})^{n}=x^{n} \bar{\theta}=x^{n} \theta=g .
$$

Lemma 2.2. Every locally nilpotent subgroup $H$ of a quasi-injective group is a Dedekind group (i.e. each subgroup of $H$ is normal in $H$ ).

Proof. It is sufficient to prove that every finitely generated nilpotent subgroup $H$ is Dedekind.

Let $K=\gamma_{3}(H)=\left[H^{\prime}, H\right]$; we show first that $H^{\prime} / K$ is finite.

Let $K \leqq U<H$; then $U \triangleleft U H^{\prime} \triangleleft H$. If $U$ is not normal in $H$ then there are elements $h \in H$ such that $U^{h} \neq U$. Suppose, if possible, that $U^{h} U / U$ is nonperiodic for some $h \in H$. Then $U^{h} U / U$ contains an infinite cyclic subgroup $C / U$ and so there is a homomorphism $\theta: C \rightarrow G$ with $\operatorname{Ker} \theta=U$. There is an extension $\bar{\theta}: G \rightarrow G$ of $\theta$ with $\operatorname{Ker} \bar{\theta}=N$, say. Then $N \triangleleft G$ and $N \cap C=U$. But $U \leqq N \triangleleft G$ implies that $U^{h} \leqq N$ and so $C \leqq U U^{h} \leqq N$. This contradiction shows that $U^{h} U / U$ is periodic for all $h \in H$. Therefore $U^{H} / H$ is periodic and, being a finitely generated nilpotent group, $U^{H} / U$ is finite. The finitely generated group $U^{H}$ has only finitely many subgroups of index $\left|U^{H}: U\right|$ and so $U$ has only finitely many conjugates in $H$.

We have shown that each subgroup of $H / K$ has only finitely many conjugates. By a theorem of Neumann [4], $H / K$ is centre-by-finite and hence is finite-by-abelian. Thus $H^{\prime} / K=\gamma_{2}(H) / \gamma_{3}(H)$ is finite.

It follows that $\gamma_{i}(H) / \gamma_{i+1}(H)$ is finite for each $i \geqq 2\left[7\right.$, p. 55] and so $H^{\prime}$ is finite.

Now we can complete the proof. If $H$ is not a Dedekind group then there is a subgroup $M<H$ such that $M \triangleleft^{2} H$ but $M \not H$. Then $M H^{\prime} \triangleleft H$ and so there is a subgroup $L$ such that $M \triangleleft L \triangleleft H$ and $L \leqq M H^{\prime}$. Since $M \Varangle H$, there is an element $h \in H$ such that $M^{h} \neq M$ so that $M<M M^{h} \leqq L \leqq M H^{\prime}$. Since $H^{\prime}$ is finite, $M M^{h} / M$ contains a cyclic subgroup $C / M$ of prime order $p$, say, where $p$ divides $\left|H^{\prime}\right|$. Then $H^{\prime}$ contains an element of order $p$ and there is a homomorphism $\phi: C \rightarrow G$ with $\operatorname{Ker} \phi=M$. This mapping has an extension $\phi: G \rightarrow G$ with $\operatorname{Ker} \phi=T$ and $T \cap C=M$. But $T \triangleleft G$ implies that $M^{h} \leqq T$ and so $C \leqq M M^{h} \leqq T$. This final contradiction shows that $H$ is a Dedekind group.

Corollary 2.3. Every normal (locally) nilpotent subgroup of a quasi-injective group is 
either abelian or is a direct product, $Q \times A$, of a quaternion group $Q$ of order 8 and a periodic abelian 2'-group.

Proof. The normal locally nilpotent subgroup $N$ is a Dedekind group and so is either abelian or a direct product $Q \times A$ of a quaternion group and a periodic abelian group [3, Satz III.7.12].

We may therefore suppose that $N=Q \times A$ and it remains to show that $A$ has no elements of order 2. Let $\langle q\rangle$ be the centre of $Q$ and let $x \in A$ be an element of order 2 . There is an isomorphism $\theta:\langle q\rangle \rightarrow\langle x\rangle$ mapping $q$ onto $x$. Let $\theta: G \rightarrow G$ be an extension of $\theta$ and let $\operatorname{Ker} \bar{\theta}=K$. Since $K \cap\langle q\rangle=1$, we must have $K \cap Q=1$ and so there is a quaternion group $Q_{1}=Q \theta$ containing $x$. Now $Q$ is characteristic in $N$ and hence normal in $G$. Thus $Q Q_{1}$ is a finite 2-group and so, by Lemma 2.2, is a Dedekind group. But such a group contains only one quaternion group and so $Q_{1}=Q$ contrary to $x \notin Q$.

Lemma 2.4 Let $N$ be a normal (locally) nilpotent subgroup of the quasi-injective group $G$. Then every subgroup of $N$ is normal in $G$.

Proof. It is sufficient to prove that each cyclic subgroup $X=\langle x\rangle$ of $N$ is normal in $G$. Suppose first that $X$ has finite order and let $g \in G$. It follows from Lemma 2.2 that $X \triangleleft N$ and so $U=X X^{g}$ is a finite nilpotent group with $X \triangleleft U$. If $X^{g} \neq X$, then $U / X$ contains a subgroup $C / X$ of prime order and there is a homomorphism $\theta: C \rightarrow G$ with $\operatorname{Ker} \theta=X$. There is an endomorphism $\bar{\theta}: G \rightarrow G$ extending $\theta$ with $\operatorname{Ker} \bar{\theta}=Y$, say. Then $Y \triangleleft G$ implies that $Y \geqq X X^{\theta}=U \geqq C$ contrary to $C \cap Y=X<C$. This contradiction shows that $X \triangleleft G$ whenever $X$ is finite.

Now suppose that $X$ is infinite cyclic; by Corollary $2.3, N$ must be abelian. Let $g \in G$ and $U=X X^{g}$; then $U$ is a finitely generated abelian group. If $U / X$ is infinite cyclic then there is a homomorphism $\phi: U \rightarrow G$ with $\operatorname{Ker} \phi=X$. This extends to an endomorphism $\Phi: G \rightarrow G$ with $\operatorname{Ker} \phi=W$, say. Then $W \triangleleft G$ implies that $W \geqq X X^{g}=U$ contrary to $U \cap W=$ $X<U$. We may therefore assume that $U / X$ is finite.

If the torsion subgroup $T$ of $U$ is non-trivial, then $T X / X \cong T$ and so $U / X$ contains a subgroup $C / X$ of prime order $p$ for some $p$ dividing $|T|$. Thus there is a homomorphism $\psi: C \rightarrow G$ with $\operatorname{Ker} \psi=X$ and we obtain a contradiction as above.

Therefore, we may assume that $U$ is torsion-free. Since $U / X$ is finite it follows that $U$ is infinite cyclic so that either $U=X$ or $U=X^{g}$. If $X^{\theta} \neq X$, then either (1) $g^{-1} x g=x^{n}$ or (2) $g^{-1} x^{n} g=x$ for some integer $n \neq 0, \pm 1$. There is a positive integer $r$ such that $n$ is not an $r$ th power. Since, by Lemma 2.1(iv), $G$ is radicable, there is an element $h \in G$ such that $h^{r}=g$. Since $X^{g} \neq X$, we also have $X^{h} \neq X$ and, as above, we can show that $X X^{h}$ is infinite cyclic and either (a) $h^{-1} x h=x^{m}$ or (b) $h^{-1} x^{m} h=x$, for some integer $m \neq 0, \pm 1$. From (a) we obtain $g^{-1} x g=h^{-r} x h^{r}=x^{m^{r}}$ so that either (1) $x^{m^{\prime}}=x^{n}$ and $m^{r}=n$ or (2) $g^{-1} x^{n} g=x^{n m^{\prime}}=x$ so that $n m^{r}=1$. From (b) we obtain $g^{-1} x^{m^{\prime}} g=h^{-r} x^{m^{\prime}} h^{r}=x$ so that either (1) $g^{-1} x^{m^{\prime}} g=x=x^{n m^{r}}$ and $n m^{r}=1$ or (2) $g^{-1} x^{m^{r}} g=x=g^{-1} x^{n} g$ and $n=m^{r}$. In all cases we have a contradiction to $n$ not being an $r$ th power and this completes our proof that $X \triangleleft G$.

Corollary 2.5. Let $G$ be a quasi-injective group. Then $C_{G}\left(G^{\prime}\right)$ is the unique maximal normal (locally) nilpotent subgroup of $G$. 
Proof. Certainty $C_{G}\left(G^{\prime}\right)$ is a normal nilpotent subgroup. If $N$ is any normal locally nilpotent subgroup and $x \in N$, then $\langle x\rangle \triangleleft G$ and so $G / C_{G}(\langle x\rangle)$ is abelian. Hence $C_{G}(\langle x\rangle) \geqq G^{\prime}$ and so $x \in C_{G}\left(G^{\prime}\right)$.

Theorem 2.6. A soluble quasi-injective group is metabelian.

Proof. If not, then since the terms of the derived series are fully invariant there is a soluble quasi-injective group $G$ of derived length 3 . In this group $G^{\prime \prime}$ is a normal abelian subgroup and so, by Corollary $2.5, G^{\prime} \leqq C_{G}\left(G^{\prime}\right)$; that is, $G^{\prime}$ is abelian and $G$ metabelian.

Theorem 2.7. A nonperiodic soluble quasi-injective group $G$ is abelian and hence is a divisible abelian group.

Proof. Let $x \in G^{\prime}$; then $\langle x\rangle \triangleleft G$ and so $G / C_{G}(\langle x\rangle)$ is finite. But, by Lemma 2.1(iv), $G$ is radicable and so has no non-trivial finite factor group. Therefore $x$ is central and hence $G^{\prime}$ is central. Therefore $G$ is nilpotent and the result now follows from Corollary 2.3 .

\section{Locally finite groups}

We have now reduced our investigation of soluble quasi-injective groups to the locally finite case. In fact, we shall see that the locally finite quasi-injective groups are necessarily soluble. Our methods in this section are much closer to those used by Bertholf and Walls [1] in the finite case although some complications arise through not being able to assume that the groups have a satisfactory theory of Sylow subgroups.

Lemma 3.1. A locally finite quasi-injective group $G$ is locally supersoluble.

Proof. Let $F$ be any finite subgroup of $G$ and let $U / V$ be a chief factor of $F$. If $U / V$ is not cyclic of prime order than there is a prime $p$ such that $U / V$ has a subgroup $E / V \cong C_{p} \times C_{p}$. (If $U / V$ is nonabelian we can take $p=2$.) There is a homomorphism $\theta: E \rightarrow G$ with $|\operatorname{Im} \theta|=p$ and $\mathrm{V}<\operatorname{Ker} \theta<E$. This mapping $\theta$ can be extended to an endomorphism $\bar{\theta}: G \rightarrow G$ with $\operatorname{Ker} \bar{\theta}=K$, say. Then $K \cap E=\operatorname{Ker} \theta$ and so $V<K \cap U<U$. But $K \cap U \triangleleft F$ and we have a contradiction to $U / V$ being a chief factor of $F$.

Corollary 3.2. A locally finite quasi-injective group is metabelian.

Proof. By Lemma 3.1, $G^{\prime}$ is locally nilpotent and, by Corollary $2.3, G^{\prime}$ is metabelian. Therefore $G$ is soluble and Theorem 2.6 shows that $G$ is metabelian.

We shall use the term Sylow p-subgroup simply to mean a maximal p-subgroup. It follows from Lemma 2.2 that a Sylow p-subgroup $S$ of a locally finite quasi-injective group is a Dedekind group. It follows that $S$ is either abelian or is the direct product of a quaternion group and an elementary abelian 2-group [3, Satz III.7.12]. We can improve this result as follows.

Lemma 3.3. Let $G$ be a locally finite quasi-injective group and let $S$ be a Sylow p- 
subgroup of $G$. If $p$ is odd then $S$ is abelian. If $p=2$ then $S$ is either abelian or $a$ quaternion group of order 8.

Proof. By the remarks above we need only consider the case in which $p=2$ and $S=Q \times E$ where $Q$ is a quaternion group and $E$ an elementary abelian 2-group.

Suppose that $E \neq 1$ and let $x$ be a non-trivial element in $E$. Let $\langle q\rangle$ be the centre of $Q$ so that there is an isomorphism $\theta:\langle q\rangle \rightarrow\langle x\rangle$ mapping $q$ onto $x$. Let $\bar{\theta}: G \rightarrow G$ be an extension of $\theta$; then $Q \cap \operatorname{Ker} \bar{\theta}=1$ and so there is a quaternion group $Q_{1}=Q \bar{\theta}$ with $\langle x\rangle$ as centre.

There is a finite subgroup $F$ containing $Q$ and $Q_{1}$. Let $T$ and $T_{1}$ be Sylow 2subgroups of $F$ containing $Q$ and $Q_{1}$, respectively. Since $T$ and $T_{1}$ are Dedekind groups, $Q$ and $Q_{1}$ are characteristic subgroups of $T$ and $T_{1}$ and hence there is an element $g \in F$ such that $Q_{1}=g Q g^{-1}$ and so $x=g q g^{-1}$. The homomorphism $\phi:\langle x, q\rangle \rightarrow\langle x\rangle$ with $\operatorname{Ker} \phi=$ $\langle q\rangle$ has an extension $\phi: G \rightarrow G$. If $M=\operatorname{Ker} \phi$, then $M \geqq\left\langle q, g q g^{-1}\right\rangle=\langle x, q\rangle$ contrary to $M \cap\langle x, q\rangle=\operatorname{Ker} \phi=\langle q\rangle$. Therefore $E=1$ and $S$ is a quaternion group.

Theorem 3.4. If the locally finite quasi-injective group $G$ has a quaternion Sylow 2subgroup $Q$, then $G=Q \times H$ where $H$ is a quasi-injective $2^{\prime}$-group.

Proof. Since $G$ has a finite Sylow 2-subgroup, the Sylow 2-subgroups of any subgroup $U$ of $G$ are conjugate in $U$. Now $Q$ is a Sylow 2-subgroup of the normal subgroup $Q G^{\prime}$ and so, by the Frattini argument, $G=Q G^{\prime} N_{G}(Q)=G^{\prime} N_{G}(Q)$. If $Q \nless G$ then there is a $2^{\prime}$-element $x \in G^{\prime}-N_{G}(Q)$. By Corollary $3.2, G^{\prime}$ is abelian and so, by Lemma $2.4,\langle x\rangle \triangleleft G$.

The natural homomorphism $\theta:\langle x\rangle C_{Q}(x) \rightarrow\langle x\rangle$ can be extended to an endomorphism $\bar{\theta}: G \rightarrow G$ with $\operatorname{Ker} \bar{\theta}=M$, say. Then $C_{Q}(x) \leqq M \cap\langle x\rangle Q \triangleleft\langle x\rangle Q$. Also $M \cap\langle x\rangle=1$ so that $[M \cap\langle x\rangle Q,\langle x\rangle] \leqq M \cap\langle x\rangle=1$ and we obtain $C_{Q}(x)=M \cap\langle x\rangle Q$. Therefore $\operatorname{Im} \bar{\theta}$ contains a subgroup isomorphic to $\langle x\rangle Q / C_{Q}(x)$.

But $Q / C_{Q}(x)$ is a non-trivial cyclic group and so has order 2. Therefore $C_{Q}(x)$ contains the centre of $Q$. Since the Sylow 2-subgroups of $G$ are conjugate to $Q$ and $\langle x\rangle \triangleleft G$ it follows that each element of order 2 centralizes $x$. However, $\langle x\rangle Q / C_{Q}(x)$ contains an element of order 2 which does not centralize $\bar{x}$. Hence $\operatorname{Im} \bar{\theta}$ contains an element of order 2 which does not centralize $x \theta=x$. This contradiction shows that $Q \triangleleft G$.

But by Lemma 3.1, $G$ is locally supersoluble and so has a normal Sylow 2'-subgroup $H$, say. Then $G=Q \times H$ and it follows from Lemma 2.1(i) that $H$ is quasi-injective.

The following lemma shows that groups of the above form are necessarily quasiinjective. This gives part (B)(I) of our main theorem and reduces the characterization of locally finite quasi-injective groups to the case in which the Sylow p-subgroups are abelian for each prime $p$.

Lemma 3.5. Let $G=S \times T$, where $S$ is a $\pi$-group and $T$ a $\pi^{\prime}$-group. Then $G$ is quasiinjective if and only if $S$ and $T$ are quasi-injective.

Proof. One direction is contained in Lemma 2.1(i). We assume therefore that $S$ and $T$ are quasi-injective groups, $H$ is a subgroup of $G$ and $\theta: H \rightarrow G$ is a homomorphism. Then there are endomorphisms $\theta_{S}: S \rightarrow S$ and $\theta_{T}: T \rightarrow T$ which extend $\left.\theta\right|_{H \cap S}$ and $\left.\theta\right|_{H \cap T}$. 
Since $H=(H \cap S) \times(H \cap T)$ it is easy to check that the mapping $\bar{\theta}: G \rightarrow G$ defined by

$$
(s t) \bar{\theta}=\left(s \theta_{S}\right)\left(t \theta_{T}\right), \quad \text { for } s \in S, \quad t \in T,
$$

is an endomorphism extending $\theta$.

The remainder of the paper is concerned with locally finite quasi-injective groups in which every $p$-subgroup is abelian for every prime $p$. We begin by proving that these groups satisfy the conditions of part (B)(II) of the main theorem (Lemmas 3.6 to 3.10) and then show that a group with these properties is quasi-injective (Theorem 3.13).

Lemma 3.6. Let $G$ be a locally finite quasi-injective group with abelian Sylow subgroups. Then $G^{\prime}$ is a maximal $\pi$-subgroup for some set of primes $\pi$, and each Sylow psubgroup of $G$ is a direct product of isomorphic cyclic or quasicyclic groups.

Proof. If $G^{\prime}$ contains elements of order $p^{n}$ for each positive integer $n$, then $G^{\prime}$ contains all $p$-elements of $G$, by Lemma 2.1(iii), and so $G^{\prime}$ contains a Sylow p-subgroup of $G$.

Suppose then that $p^{n}$ is the maximal order of a p-element in $G^{\prime}$, where $n \geqq 1$. Then $G^{\prime}$ contains all elements of order dividing $p^{n}$. If $G^{\prime}$ does not contain a Sylow $p$-subgroup of $G$ then there is an element $a \notin G^{\prime}$ such that $a$ has order $p^{n+1}$. There is a finite subgroup $F$ such that $a \in F$ and $a^{p} \in F^{\prime}$. Let $P$ be a Sylow p-subgroup of $F$ containing $a$. Since $P$ is abelian we have, by Satz III.13.4 in [3],

$$
P=\left(P \cap F^{\prime}\right) \times\left(P \cap Z\left(N_{F}(P)\right)\right) .
$$

Write $a=x y$, with $x \in P \cap F^{\prime}$ and $y \in P \cap Z\left(N_{F}(P)\right)$ : then $a^{p}=x^{p} y^{p} \in P \cap F^{\prime}$ and so $y^{p}=1$. Hence $a^{p}=x^{p}$ and $x$ is an element of $G^{\prime}$ of order $p^{n+1}$. This contradiction shows that the Sylow p-subgroup of $G^{\prime}$ is a Sylow $p$-subgroup of $G$.

The structure of the Sylow $p$-subgroup $S$ of $G$, for $p \in \tilde{\omega}\left(G^{\prime}\right)$, follows from the observation that any two elements $x, y$ of order $p$ have the same height in $S$. For, suppose $x$ has height $n$ in $S$ and $y$ has height greater than $n$. Then there is an element $g \in S$ such that $g^{m^{n+1}}=y$. The isomorphism $\theta:\langle y\rangle \rightarrow\langle x\rangle$ mapping $y$ onto $x$ extends to an endomorphism $\bar{\theta}: G \rightarrow G$. Clearly $(g \bar{\theta})^{p^{n+1}}=x$ and $g \bar{\theta}$, being a $p$-element, is an element of the normal Sylow $p$-subgroup $S$.

By Lemma 2.4, every subgroup of $G^{\prime}$ is normal in $G$ and so each element of $G$ induces an automorphism of the abelian group $G^{\prime}$ which fixes each subgroup. Robinson [6] calls such a subgroup-preserving automorphism a power automorphism. We restate the relevant part of his Lemma 4.1.1 on power automorphisms in the context of quasiinjective groups.

Lemma 3.7. Let $G$ be a locally finite quasi-injective group with abelian Sylow subgroups and let $g \in G$. Then, for each prime $p \in \bar{\omega}\left(G^{\prime}\right)$ and each positive integer $r$, there is an integer $m=m(p, r, g)$ such that $g^{-1} a g=a^{m}$, for every element $a \in G^{\prime}$ of order dividing $p^{\prime}$.

Lemma 3.8. Let $G$ be a locally finite quasi-injective group with abelian Sylow subgroups and let $\pi=\tilde{\omega}(G)-\tilde{\omega}\left(G^{\prime}\right)$. Then each maximal $\pi$-subgroup of $G$ is a complement to $G^{\prime}$. 
Proof. Note first that $G^{\prime} \cap Z(G)=1$. For, otherwise there is a $p$-element $x \in G^{\prime} \cap Z(G)$. There is a finite subgroup $F$ of $G$ such that $x \in F^{\prime}$ and $F$ has an abelian normal Sylow $p$ subgroup $P$, say. Then $x \in P \cap F^{\prime} \cap Z(F)$ contrary to this subgroup being trivial, by Satz IV.2.2 in [3].

Now let $C=C_{G}\left(G^{\prime}\right)$ the maximal normal locally nilpotent subgroup of $G$ (Corollary 2.5). Then $C=G^{\prime} \times D$, where $D$ is an abelian normal $\pi$-subgroup of $G$. Since $[G, D] \leqq G^{\prime} \cap D=1$ we see that $D=Z(G)$.

For each $p \in \tilde{\omega}\left(G^{\prime}\right)$, let $C_{p}=C_{G}\left(\left(G^{\prime}\right)_{p}\right)$. It follows from Lemma 3.7 that $G / C_{p}$ is finite and so there is a countable subgroup $L / C$ of $G / C$ such that $L C_{p}=G$, for all $p \in \tilde{\omega}\left(G^{\prime}\right)$. Then $L / D$ is an extension of the normal $\pi^{\prime}$-subgroup $C / D$ by a countable $\pi$-group and so $C / D$ has a complement $K / D$ in $L / D$. Now $K$ is a $\pi$-group, $K / D$ is countable and $K C_{p}=G$, for all $p \in \tilde{\omega}\left(G^{\prime}\right)$. We show first that $C_{G}(K)$ is a complement to $G^{\prime}$ in $G$.

If $G^{\prime} \cap C_{G}(K) \neq 1$, then there is a $p$-element $x \in G^{\prime} \cap C_{G}(K)$. The element $x$ centralizes $C_{p}$ and $K$ and hence $x \in Z(G) \cap G^{\prime}=1$. Thus $G^{\prime} \cap C_{G}(K)=1$ and, in particular, $C_{G}(K)$ is an abelian $\pi$-group.

Let $g \in G$ and $H=K G^{\prime}\langle g\rangle$ so that $H / C$ is a countable $\pi$-group. Then $C / D$ has a complement $S / D$ in $H / D$. Since $D=Z(G), S$ is a $\pi$-group and $S G^{\prime}=H$. It follows that $S \cap K G^{\prime}$ and $K$ are both complements to $G^{\prime}$ in $K G^{\prime}$. Since $G^{\prime}$ is abelian, we may apply Hilfssatz VI.7.14 of [3] to obtain an automorphism $\theta$ of $K G^{\prime}$ which coincides with the identity on $G^{\prime}$, maps $S \cap K G^{\prime}$ isomorphically onto $K$ and satisfies $(x \theta) x^{-1} \in G^{\prime}$, for all $x \in K G^{\prime}$. There is an endomorphism $\theta: G \rightarrow G$ which coincides with $\theta$ on $K G^{\prime}$. Suppose that $S=\left(S \cap K G^{\prime}\right)\langle y\rangle$, so that $H=K G^{\prime}\langle y\rangle$. Since $\bar{\theta}$ coincides with the identity on $G^{\prime}$, we have $(y \bar{\theta}) y^{-1} \in C \leqq K G^{\prime}$. Therefore $y \in K G^{\prime}(y \bar{\theta}) \subseteq K G^{\prime}(S \bar{\theta})$. But $S \bar{\theta}$ is a $\pi$-group containing $K$ and so $S \bar{\theta} \leqq C_{G}(K)$. Therefore $y \in G^{\prime} C_{G}(K)$. Hence $g \in H=S G^{\prime}\langle y\rangle \leqq G^{\prime} C_{G}(K)$ and, since $g$ was chosen arbitrarily, we have $G^{\prime} C_{G}(K)=G$ and so $C_{G}(K)$ is a complement to $G^{\prime}$.

Now let $T$ be a complement to $G^{\prime}$ (e.g., $T=C_{G}(K)$ ) and let $U$ be any maximal $\pi$ subgroup of $G$. Then $U$ and $T \cap U G^{\prime}$ are complements to $G^{\prime}$ in $U G^{\prime}$. As above there is an automorphism $\phi$ of $U G^{\prime}$ coinciding with the identity on $G^{\prime}$ and mapping $T \cap U G^{\prime}$ isomorphically onto $U$. Let $\phi: G \rightarrow G$ be an endomorphism extending $\phi$. Then $T \bar{\phi}$ is a $\pi$ group containing $U$ and by the maximality of $U$ we have $T \phi=U$. If $U G^{\prime} \neq G$, then since $\left(T \cap U G^{\prime}\right) \bar{\phi}=T \bar{\phi}$ we obtain $T \cap \operatorname{Ker} \phi \neq 1$. But

$$
[T \cap \operatorname{Ker} \phi, G] \leqq \operatorname{Ker} \phi \cap G^{\prime}=1
$$

and so $T \cap \operatorname{Ker} \phi$ is a central $\pi$-subgroup. Hence $T \cap \operatorname{Ker} \phi$ is contained in the maximal $\pi$-subgroup $U$, contrary to $T \cap U G^{\prime} \cap \operatorname{Ker} \phi=1$.

Lemma 3.9. Let $G$ be a locally finite quasi-injective group with abelian Sylow subgroups. If $S$ is a Sylow p-subgroup of $G$, then $S$ is the direct product of isomorphic cyclic or quasicyclic subgroups.

Proof. This has already been proved in Lemma 3.6 for primes $p \in \tilde{\omega}\left(G^{\prime}\right)$. For primes $p \notin \tilde{\omega}\left(G^{\prime}\right)$ we require the complementation result proved above. As in Lemma 3.6 we can prove that any two elements of order $p$ have the same $p$-height in $G$. We must show that an element $x \in S$ of order $p$ has the same $p$-height in $S$ as in $G$. Suppose that $y$ is a 
p-element such that $x \in\langle y\rangle$; we must show that there is an element $g \in S$ having the same order as $y$ such that $x \in\langle g\rangle$. There is a Sylow p-subgroup $P$ of $G$ containing $y$ and, by Lemma 3.8 and Hilfssatz VI.7.14 of [3], there is an automorphism $\phi$ of $G$ such that $P \phi=S$ and $\phi$ induces the identity on $G / G^{\prime}$. Then $x \phi \in\langle y \phi\rangle \leqq S$ and $x \phi \in G^{\prime}\langle x\rangle$. But $S \cap G^{\prime}\langle x\rangle=\langle x\rangle$ and it follows that $x \phi=x$. Therefore $x \in\langle y \phi\rangle \leqq S$ and $x$ has the same $p$ height in $S$ as in $G$.

Lemma 3.10. Let $G$ be a locally finite quasi-injective group with abelian Sylow subgroups. Let $\pi=\tilde{\omega}(G)-\tilde{\omega}\left(G^{\prime}\right), H$ a maximal $\pi$-subgroup of $G$ and $G_{\sigma}^{\prime}$ a maximal $\sigma$ subgroup of $G^{\prime}$, for some set of primes $\sigma \subseteq \tilde{\omega}\left(G^{\prime}\right)$. Then $C_{H}\left(G_{\sigma}^{\prime}\right)$ is a direct factor of $H$. In particular, $Z(G)=C_{H}\left(G^{\prime}\right)$ is a direct factor of $H$.

Proof. It is sufficient to prove that if $h \in H$ such that $h^{p} \in C_{H}\left(G_{\sigma}^{\prime}\right)$ for some prime $p \in \pi$, then there is an $x \in C_{H}\left(G_{\sigma}^{\prime}\right)$ such that $h^{p}=x^{p}$.

The natural homomorphism $\theta: G_{\sigma}^{\prime}\left\langle h^{p}\right\rangle \rightarrow G_{\sigma}^{\prime}$ can be extended to an endomorphism $\bar{\theta}: G \rightarrow G$ with $\operatorname{Ker} \bar{\theta}=M$, say. Since $h \notin C_{H}\left(G_{\sigma}^{\prime}\right)$, we have $M \cap G_{\sigma}^{\prime}\langle h\rangle=\left\langle h^{p}\right\rangle$ and so $\operatorname{Im} \bar{\theta}$ contains an element $y$ with the same action on $G_{\sigma}^{\prime}$ as $h$. Now $y \in G^{\prime}\langle y\rangle=G^{\prime}\left(G^{\prime}\langle y\rangle \cap H\right)$ and $G^{\prime}\langle y\rangle \cap H$ is a finite Sylow $\pi$-subgroup of $G^{\prime}\langle y\rangle$. Therefore there is an element $g \in G^{\prime}$ such that $\langle y\rangle^{g} \leqq H$. Clearly $y^{g}$ still has the same action on $G_{\sigma}^{\prime}$ as $h$ and so $x=$ $h\left(y^{g}\right)^{-1} \in C_{H}\left(G_{\sigma}^{\prime}\right)$ is the required element.

We now show that a group with the properties obtained in Lemmas 3.6 to 3.10 is in fact a quasi-injective group.

Theorem 3.11. Let $G$ be a split extension of a normal $\pi^{\prime}$-group $K$ by a $\pi$-group $H$ satisfying the following conditions:

(i) $H$ and $K$ are abelian groups in which each Sylow subgroup is a direct product of isomorphic cyclic or quasicyclic groups,

(ii) $K=G^{\prime}$,

(iii) every maximal $\pi$-subgroup of $G$ is a complement to $K$,

(iv) for each $h \in H, p \in \pi^{\prime}$ and positive integer $r$, there is an integer $m=m(p, r, h)$ such that $h^{-1} a h=a^{m}$, for every element $a \in K$ of order dividing $p^{r}$,

(v) if $\sigma \subseteq \pi$ and $K_{\sigma}$ is the Sylow $\sigma$-subgroup of $K$, then $C_{H}\left(K_{\sigma}\right)$ is a direct factor of $H$.

Then $G$ is a quasi-injective group.

Proof. Let $L \geqq G$ and let $\theta: L \rightarrow G$ be a homomorphism.

(A) If $\sigma=\tilde{\omega}(K \cap L / K \cap L \cap \operatorname{Ker} \theta)$ and $x \in L$, then $x^{-1}(x \theta) \in C_{G}\left(K_{\sigma}\right)$.

Let $p \in \sigma$ and $k \in\left(K_{p} \cap L\right)-\operatorname{Ker} \theta$. Then, using (iv), we obtain $(k \theta)^{x \theta}=\left(k^{x}\right)^{\theta}=(k \theta)^{x}$. Thus $x^{-1}(x \theta) \in C_{G}(k \theta)$. But, using (iv) again, $C_{G}(k \theta)=C_{G}\left(K_{p}\right)$. Therefore $x^{-1}(x \theta)$ centralises $K_{p}$ for all $p \in \sigma$ and so $x^{-1}(x \theta) \in C_{G}\left(K_{\sigma}\right)$.

(B) The homomorphism $\theta: L \rightarrow G$ can be extended to a homomorphism $\theta_{1}: K L \rightarrow G$ such that $K_{\sigma^{\prime}} \leqq \operatorname{Ker} \theta_{1}$.

Since $K$ is a quasi-injective abelian group and $(K \cap L) \theta \leqq K_{o}$, we can extend $\left.\theta\right|_{K \cap L}$ to a 
homomorphism $\theta_{2}: K \rightarrow K$ such that $K_{\sigma} \theta_{2} \leqq K_{\sigma}$ and $K_{\sigma^{\prime}} \leqq \operatorname{Ker} \theta_{2}$. We now define $\theta_{1}: K L \rightarrow G$ by

$$
(k x) \theta_{1}=\left(k \theta_{2}\right)(x \theta), \quad \text { for } k \in K, \quad x \in L .
$$

Since $\theta_{2}$ is an extension of $\left.\theta\right|_{K \cap L}, \theta_{1}$ is well-defined. Also $\theta_{1}$ is a homomorphism, since

$$
\begin{aligned}
\left(k_{1} x_{1}\right) \theta_{1}\left(k_{2} x_{2}\right) \theta_{1} & =\left(k_{1} \theta_{2}\right)\left(x_{1} \theta\right)\left(k_{2} \theta_{2}\right)\left(x_{2} \theta\right) \\
& =\left(k_{1} \theta_{2}\right)\left(k_{2} \theta_{2}\right)^{\left(x_{1} \theta\right)^{-1}}\left(x_{1} \theta\right)\left(x_{2} \theta\right) \\
& =\left(k_{1} \theta_{2}\right)\left(k_{2} \theta_{2}\right)^{x_{1}^{-1}}\left(x_{1} x_{2}\right) \theta, \quad \text { by (A), } \\
& =\left(k_{1} \theta_{2}\right)\left(k_{2}^{x_{1}^{-1}}\right) \theta_{2}\left(x_{1} x_{2}\right) \theta, \quad \text { using (iv), } \\
& =\left(k_{1} k_{2}^{x_{1}^{-1}}\right) \theta_{2}\left(x_{1} x_{2}\right) \theta \\
& =\left(k_{1} x_{1} k_{2} x_{2}\right) \theta_{1} .
\end{aligned}
$$

(C) The homomorphism $\theta_{1}: K L \rightarrow G$ can be extended to $\bar{\theta}: G \rightarrow G$.

There is an ascending series from $K L$ to $G$ in which each factor is cyclic of prime order. It is sufficient to extend $\theta_{2}$ to $\theta: M \rightarrow G$ where $|M / K L|=p$ and proceed inductively as there is no problem at limit ordinals.

Now $M=K(M \cap H)$ and $K L=K(K L \cap H)$. Let $h \in(M \cap H)-K L$ be a $p$-element. Since $(K L \cap H) \theta_{1}$ is a $\pi$-group, there is an automorphism $\phi$ of $G$ coinciding with the identity on $K$ and such that $(K L \cap H) \theta_{1} \leqq H \phi$. Then $\theta_{1} \phi^{-1}: K L \rightarrow G$ is a homomorphism such that $(K L \cap H) \theta_{1} \phi^{-1} \leqq H$ and $\tilde{\omega}\left(K / K \cap \operatorname{Ker} \theta_{1} \phi^{-1}\right)=\sigma$. By $(\mathrm{A}),\left(h^{p}\right)^{-1}\left(h^{p} \theta_{1} \phi^{-1}\right) \in C_{H}\left(K_{\sigma}\right)$ or $h^{p} \theta_{1}=\left(h^{p} c\right) \phi$, for some $p$-element $c=\left(h^{-1}\left(h \theta_{1} \phi^{-1}\right)\right)^{p} \in C_{H}\left(K_{\sigma}\right)$. By $(v)$, there is an element $d \in C_{H}\left(K_{\sigma}\right)$ such that $d^{p}=c$ and so $\left(h^{p}\right) \theta_{1}=\left((h d)^{p}\right) \phi$. We can extend $\theta_{1}$ by defining $h \bar{\theta}=(h d) \phi$.

This completes the proof of our main theorem. We stated in the Introduction that soluble or locally finite quasi-injective groups are T-groups. We have not yet proved this as we have only needed the subgroups of $G^{\prime}$ to be normal in $G$. Since nonperiodic soluble groups are abelian they are clearly T-groups. For the locally finite case we can give a direct proof as in [1].

Theorem 3.12. Every subgroup of a periodic quasi-injective group is a T-group.

Proof. Suppose that $K \triangleleft L \triangleleft H \leqq G$. If $K \not H$ then there is an element $h \in H$ such that $K^{h} \neq K$. Let $U=K^{h} K \leqq L$. Since $U>K$, there is a subgroup $C / K$ of prime order $p$ and a homomorphism $\theta: C \rightarrow G$ with $\operatorname{Ker} \theta=K$. This mapping can be extended to an endomorphism $\bar{\theta}: G \rightarrow G$ with $\operatorname{Ker} \bar{\theta}=M$, say. Then $K \leqq M \triangleleft G$ and so $U=K K^{h} \leqq M$, contrary to $M \cap C=K$. 


\section{REFERENCES}

1. D. Bertholf and G. WALls, Finite quasi-injective groups, Glasgow Math. J. 20 (1979), 29-33.

2. L. Fuchs, Infinite Abelian Groups I (Academic Press, 1970).

3. B. HUPPERT, Endliche Gruppen $I$ (Grundlehren der math. Wiss. 134, Springer, 1967).

4. B. H. Neumann, Groups with finite classes of conjugate subgroups, Math. Z. 63 (1955), 7696.

5. A. Yu. Ou'Sanskil, An infinite group whose subgroups are all of prime order, Izv. Akad. Nauk SSSR Ser. Mat. 44 (1980), 309-321.

6. D. J. S. Robinson, Groups in which normality is a transitive relation, Proc. Cambridge Philos. Soc. 60 (1964), 21-38.

7. D. J. S. Robinson, Finiteness Conditions and Generalized Soluble Groups, Part I (Ergebnisse der Math. 62, Springer, 1972).

Department of Mathematics

UNIVERSITY OF GLASGOW

Glasgow G12 8QW 